\title{
Turbulence inside a vortex: Take two
}

\author{
Aline J. Cotel ${ }^{\mathrm{a})}$ \\ Department of Civil and Environmental Engineering, University of Michigan, Ann Arbor, \\ Michigan 48109-2125
}

(Received 22 March 2002; accepted 15 May 2002; published 8 July 2002)

In this paper, a modified theory based on earlier work on stratified entrainment is proposed to explain the persistence of trailing vortices in the far field. These vortices grow at a laminar rate even though the Reynolds number is high and in the turbulent regime. (C) 2002 American Institute of Physics. [DOI: 10.1063/1.1491245]

The evolution of aircraft trailing vortices is an important issue to consider while determining the minimum delay between takeoffs, especially for large busy airports, when seconds can save millions of dollars. The intensity of the trailing vortices can remain great even at large downstream distances behind lifting airplanes. Presently, a minimum delay of two minutes is used between takeoffs. However, as can be demonstrated by recent crashes, this requirement might not be sufficient to allow the vortices to dissipate.

One important aspect of the vortex evolution is the growth rate of the size of the vortex core. Previous researchers have concluded that the vortices grow at a laminar rate. This is surprising, since the Reynolds number is so high that turbulent flow is anticipated. Consequently, a turbulent growth rate would be expected. Nonetheless, data seem to show that the growth rate of trailing vortices exhibits a laminar behavior, i.e., is proportional to $\mathrm{Re}^{-1 / 2}$.

In this paper, a modified theory based on earlier work on stratified entrainment ${ }^{1}$ is proposed to explain the persistence of trailing vortices in the far field.

The previous model for the growth rate of a vortex core was based on the analogy between rotating and stratified flows. ${ }^{2}$ Bradshaw's definition of the local Richardson number is the following:

$$
\mathrm{Ri}=2 S(S+1),
$$

with $S$, the shape parameter

$$
S=(U / r)(d U / d r) .
$$

This Richardson number represents the local strength of an analogous stratification in rotating flows.

Applying this definition of Ri to a turbulent line vortex with a mean azimuthal velocity profile, we obtained the following behavior. As the radius increases, the Richardson number also increases up from 4 at $r=0$ to infinity at $r$ $=a$, equal to the vortex core radius. For $r>a$, the Richardson number decreases rapidly down to a value of zero outside the vortex (Fig. 1).

However, as pointed out by Holzapfel et al., ${ }^{3}$ Bradshaw $^{2}$ made a slight error in the definition of the Richardson num-

a) Telephone: 734-763-1463; fax: 734-763-2275. Electronic mail: acotel@engin.umich.edu ber. Some of the quantities were taken with respect to an inertial frame while others with respect to a natural coordinate frame.

If both numerator and denominator are expressed in inertial coordinates, the Richardson number becomes

$$
\mathrm{Ri}=\frac{2 \nu_{\theta}}{r^{2}} \frac{\partial \nu_{\theta} r}{\partial r} /\left(r \frac{\partial \nu_{\theta} / r}{\partial r}\right)^{2} .
$$

For a Lamb-Oseen vortex (shown to be a reasonable repre-

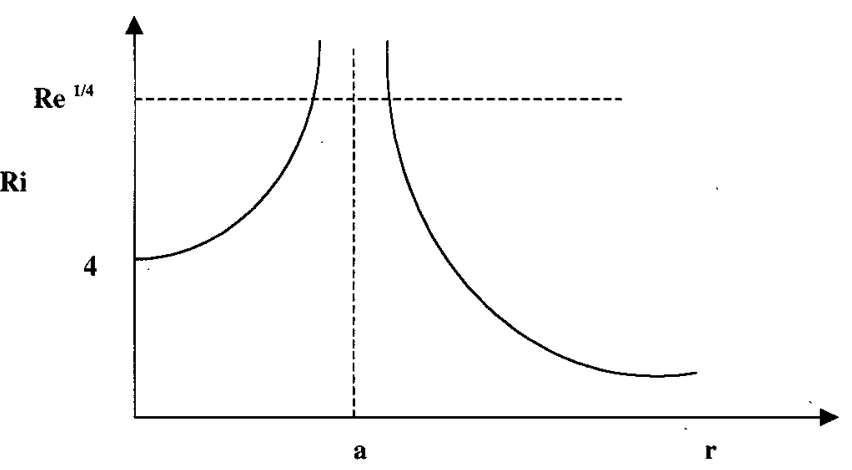

FIG. 1. Sketch of Richardson number as a function of vortex radius.

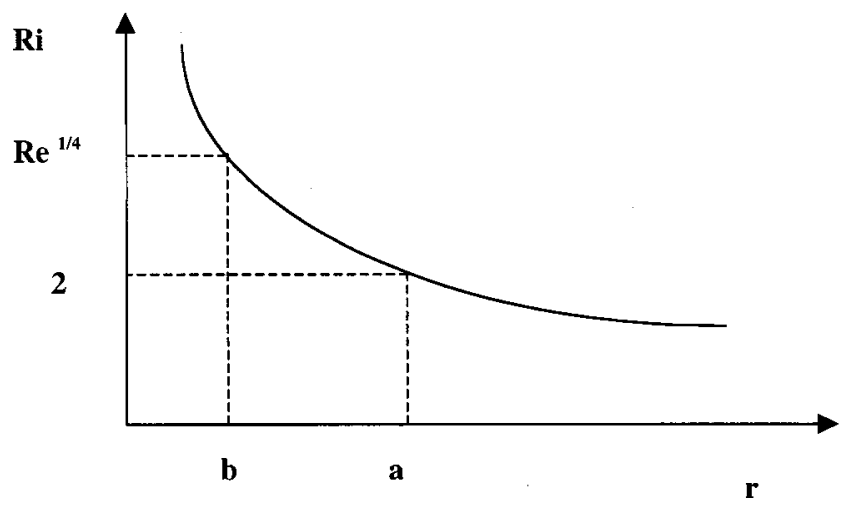

FIG. 2. Sketch of Richardson number as a function of vortex radius with the corrected Richardson number definition. 
sentation of airplane trailing vortices), ${ }^{3}$ the Richardson number goes to infinity at $r=0$, decreases as $r$ increases, reaching a value of 2 at $r=a$ (Fig. 2).

For all $\mathrm{Ri}>R e^{1 / 2}$, the flux is laminar, according to the stratified entrainment model. ${ }^{1}$ Thus at flight Reynolds numbers, this implies a strong stratification at all radii less than that corresponding to $\mathrm{Ri}=R e^{1 / 2}$ (Fig. 2). The momentum flux would be laminar for $r<b$.

The previous conclusions ${ }^{1}$ remain valid. The correction only implies a change in the location of intense stratification, i.e., large Richardson number. At $r=0$, the surface is smooth, even the Kolmogorov eddies are unable to engulf fluid from the surface. Diffusion becomes the only mechanism available for transport of momentum. This behavior is in agreement with observations. ${ }^{4}$ Turbulent vortices can exhibit laminar fluxes characteristics. This explains the persistence and strength of airplane trailing vortices in the far field. Their dissipation rate follows a laminar flow, i.e., much slower than the anticipated turbulent and more dissipative behavior.

${ }^{1}$ A. J. Cotel and R. E. Breidenthal, "Turbulence inside a vortex," Phys. Fluids 11, 3026 (1999).

${ }^{2} \mathrm{P}$. Bradshaw, "The analogy between streamline curvature and buoyancy in turbulent shear flow," J. Fluid Mech. 36, 177 (1969).

${ }^{3}$ F. Holzäpfel, T. Hofbauer, T. Gerz, and U. Schumann, "Aircraft wake vortex evolution and decay in idealized and real environments: Methodologies, benefits and imitations," Proceedings of Euromech Colloquium, 2001.

${ }^{4}$ P. Spalart, "Airplane trailing vortices," Annu. Rev. Fluid Mech. 30, 107 (1998). 